\title{
Subcortical arteriosclerotic encephalopathy (Binswanger's type) and cortical infarcts in a young normotensive patient
}

\author{
LA LOIZOU, JM JEFFERSON, W THOMAS SMITH
}

From the Department of Neurology, Queen Elizabeth Hospital, and the Department of Pathology, Medical School, Birmingham, UK

SUMMARY A 49-year-old normotensive man died after a series of strokes, slowly evolving dementia and personality change occurring over a period of 23 years. CT scan showed large infarcts involving the cortex and white matter of the temporo-occipital areas, small subcortical infarcts and low attenuation in the white matter of the frontal and parietal lobes. Neuropathological examination revealed large cortical and small subcortical infarcts corresponding to the radiological findings as well as degeneration/demyelination of central white matter corresponding to the areas of low attenuation seen on CT. The basic underlying pathological process was hyaline arteriosclerosis and atheroma which diffusely affected the small intracerebral arteries and to a lesser extent the arteries of the circle of Willis. Though unusual because of the absence of hypertension, the very early age at onset of the syndrome and the presence of large cortical infarcts this case illustrates the clinical, radiological and neuropathological features of subcortical arteriosclerotic encephalopathy (Binswanger's type).

Until recently, subcortical arteriosclerotic encephalopathy, Binswanger's type, ${ }^{1}$ had not received significant clinical and neuropathological attention in Britain. 2-4 Following Binswanger's initial description of "encephalitis subcorticalis chronica progressiva" 15 in eight patients, and further contributions by other authors, ${ }^{6-10}$ a description emerged of a syndrome consisting of slowly evolving dementia, focal neurological deficits, psychiatric disturbances, age at onset around 50 years and evidence of hypertension or arterial disease. Most recent contributions have sought to clarify the pathogenesis of this disorder and the selective vulnerability of the arterial circulation to the central white matter, ${ }^{11-14}$ while other authors have presented clinical and radiological data with which the diagnosis may be made during life. ${ }^{215-17}$ A normotensive man is described who developed subcortical arteriosclerotic encephalopathy at a young age and

Address for reprint requests: Dr LA Loizou, Midland Centre for Neurosurgery and Neurology, Holly Lane, Smethwick, Warley, West Midlands B67 7JX, UK.

Received 23 September 1981 and in revised form 26 November 1981

Accepted 12 December 1981 in whom it was possible to correlate the clinical and radiological features with pathological findings.

Case report

This man first presented in 1956 at the age of $26 \mathrm{yr}$, with a 24 hour history of headache, vomiting, mild pyrexia, progressive drowsiness, dysphasia and right hemiplegia. The blood pressure was $130 / 90 \mathrm{mmHg}$. Cerebrospinal fluid (CSF) examination showed six white cells and normal protein. Biochemical and haematological tests including serological tests for syphilis were normal. Left carotid angiogram and air ventriculogram were also normal. He made a spontaneous full recovery within three weeks. In 1963 (aged 33) he was admitted to the Neurological Unit, Queen Elizabeth Hospital, with an episode of blurring of vision, dysphasia, dyslexia and right hemiparesis, lasting 3 weeks. There were no other abnormal systemic signs, blood pressure was $140 / 95$ $\mathrm{mmHg}$. Relevant investigations included an electroencephalogram (EEG) which showed slight theta activity over the left hemisphere, an air encephalogram, which showed mild dilatation of the left lateral ventricle, and normal CSF. Other tests were non-contributory; angiography was not carried out. In the family history, his mother and two aunts had died at a young age through cerebrovascular disease, and a young cousin was said to have suffered a stroke.

A year later he was admitted to another hospital with 
a transient left hemiparesis, following chest pain diagnosed as myocardial infarction. He was anticoagulated for several months.

Over the next eight years he suffered frequent episodes of dysarthria, confusion and impairment of consciousness. In 1974 he was admitted to another hospital with a 3 day history of vomiting and dizziness. A technetium isotope brain scan was normal and the EEG showed mild nonspecific changes. The diagnosis of epilepsy was made and he was treated with carbamazepine. He then began to show changes in mood and verbal and physical aggression towards his wife; he became intensely jealous of her, and accused her of infidelity. By 1976, his memory and intellect deteriorated to the extent that he could no longer work as a machine operator.

In 1976 (aged 46) he suffered another acute episode of right hemiparesis, headache, vomiting, impairment of consciousness and subsequent confusion. Initial investigations at another hospital showed normal CSF and technetium brain scan. His recovery was slow, and it was thought that his behaviour was hysterical. On transfer to the Queen Elizabeth Hospital a month later, he was disorientated in time and place, showed impairment of both long-term and short-term memory, was unable to give the names of his wife and son, and could not tell his occupation. In addition he had mild motor dysphasia, dyslexia, dysgraphia, dyscalculia and a mild right sensory and motor deficit. Blood pressure was 125/90 $\mathrm{mmHg}$ and the systemic examination was normal. The EEG showed delta activity over the left temporal region. The CT scan showed white matter low attenuation in relation to the frontal horns, bodies of the lateral ventricles and centrum semiovale as well as small infarcts in the basal ganglia and adjacent white matter, atrophy in the region of the right sylvian fissure and low attenuation in the left occipital region consistent with cortical and subcortical infarction (fig 1). He improved except for the dementia, and was treated with oral anticoagulants and carbamazepine. $\mathbf{A}$ few months later he was assessed at the Psychiatric Unit, because of his morbid jealousy towards his wife. Psychometric testing revealed a verbal IQ of 69 , performance IQ of 52 and full scale IQ of 64 on the Wechsler Adult Intelligence Scale. On the Benton visual retention test he scored 2 (with an expected score of 4 ) and made 18 errors (expected, 8 ). He did not cooperate fully for the tests, especially on items involving execution of motor tasks, and complained of poor eyesight, although a field defect had not been detected clinically during his earlier admission.

In March 1978, he was readmitted to the Queen Elizabeth Hospital, with the history that 10 days previously he had lost consciousness at home, preceded by vertigo and headache, and followed by confusion and blindness. On examination he showed variable affect, often violent and aggressive. He was uncooperative, disorientated in place and time, denied visual and other deficits, except that at times he would say that his "memory had all gone". He showed cortical blindness and could not identify objects placed in his hands, referring to them as "keyring". There was no focal deficit in the limbs, gait was normal and blood pressure was $140 / 90 \mathrm{mmHg}$. The CT scan (fig 2) showed moderate
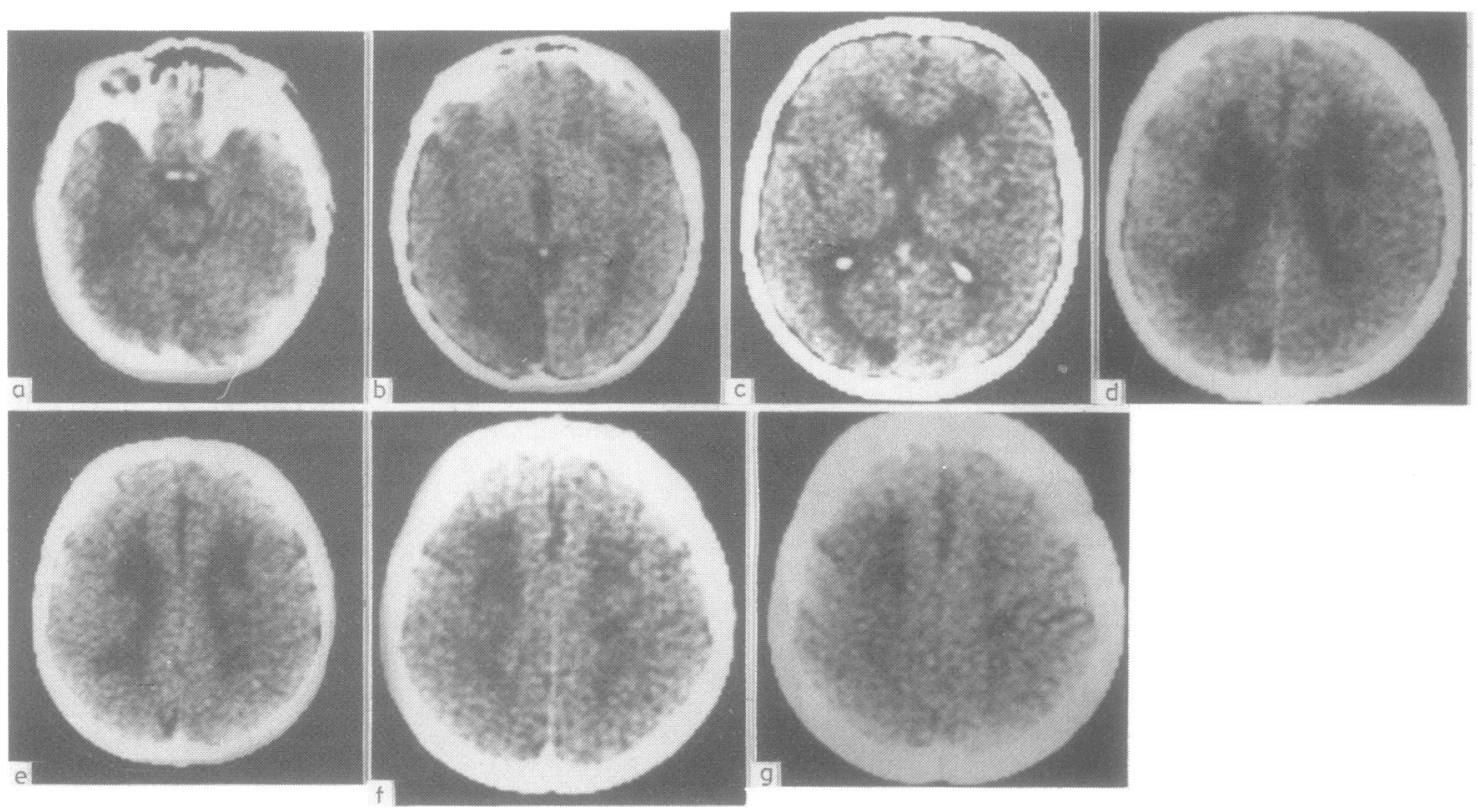

Fig 1 Initial CT scan showing white matter low attenuation in periventricular frontal and parietal white matter and centrum semiovale $(c-g)$. There are bilateral infarcts in the basal ganglia and adjocent white matter $(d)$ some focal atrophy in the right temporal lobe/insular cortex (c) and partial left occipital lobe infarction (a-c). There is no contrast enhancement $(c, d, f)$. The ventricles are slightly dilated, but cerebral cortical atrophy is very mild 

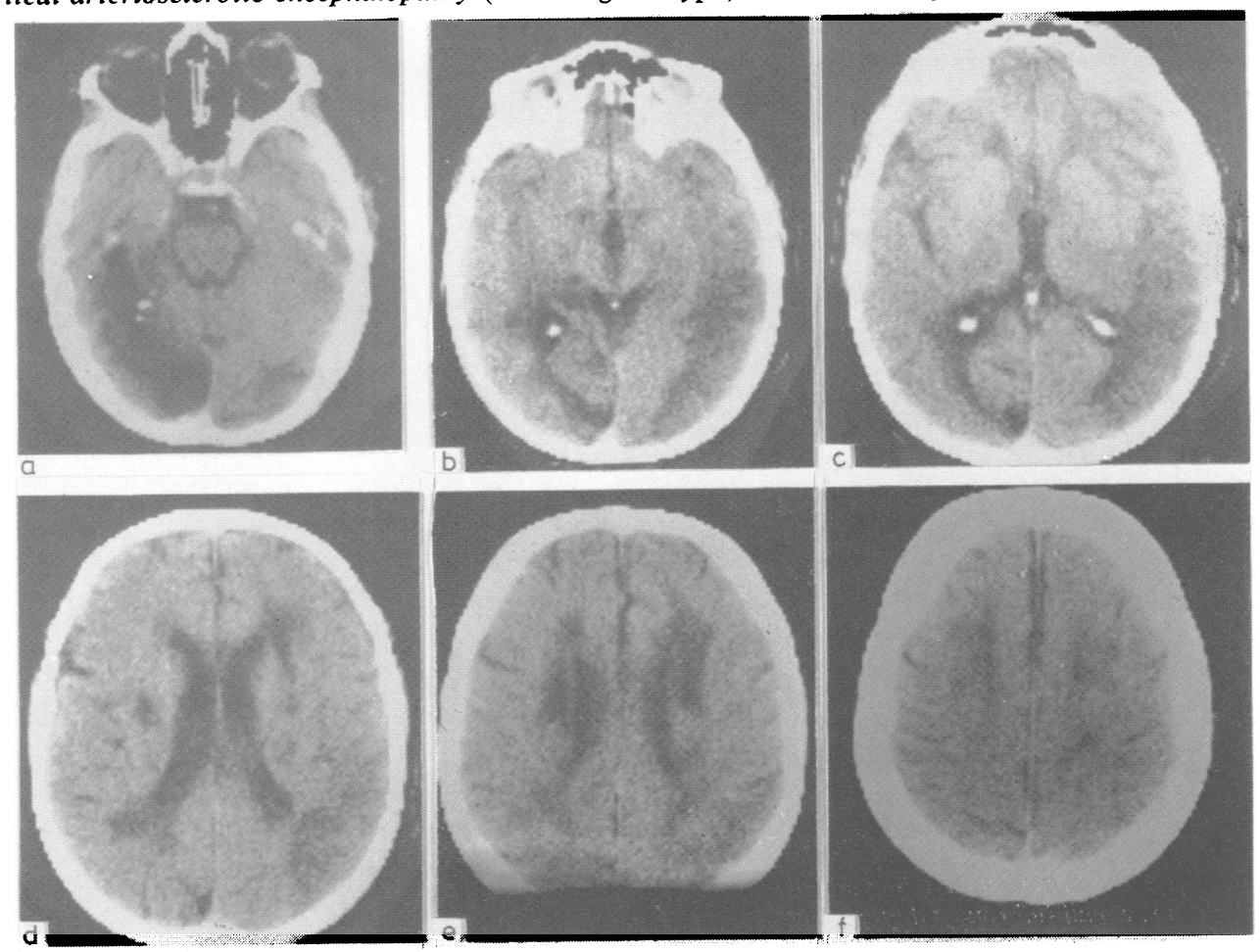

Fig 2 Second CT scan (contrast enhanced) shows an old left temporo-occipital lobe infarct (a-c) widened Sylvian fissures owing to atrophy of the temporal lobes (c) a recent right temporo-occipital infarct $(a-d)$ also demonstrated by isotope brain scan; small infarcts in the left thalamus (c), left internal capsule/caudatolenticular grey substance $(d)$, along the lateral border of the right caudate nucleus and two further infarcts in the right caudatolenticular grey matter and internal capsule $(d)$. There is white matter low attenuation around the anterior horns, bodies and posterior horns of the lateral ventricles $(d, e)$ and centrum semiovale $(f)$. There is ventricular dilatation, but only mild cerebral cortical atrophy outside the temporal lobes.

ventricular dilatation, low attenuation in the central white matter, a recent infarct in the right temporooccipital region confirmed on isotope brain scan, small subcortical infarcts and an extensive old left temporooccipital infarct. Screening investigations including EEG were normal; the ECG showed an old myocardial infarct. He was allowed home on carbamazepine, three weeks later.

He was readmitted four months later having had a seizure at home. It was impossible for his family and district nurse to manage him at home; he stayed in hospital for the rest of his life, during which time he remained demented, noisy, aggressive, incontinent and indulged in sexual activities. At times, however, he was lucid and could hold a simple conversation. He was cortically blind and had a mild dysarthria and right hemiparesis and ataxic gait. He resisted mobilisation and spent most of the time in a chair or his bed. He died of bronchopneumonia a year later at the age of 49 years.

\section{Pathological findings}

The patient was emaciated, weight $38 \mathrm{~kg}$, height $165 \mathrm{~cm}$.
There were no other external abnormalities. The heart weighed $200 \mathrm{~g}$ and there was no left ventricular hypertrophy or other evidence of systemic hypertension. The coronary arteries showed well-marked atheroma and there was a small area of fibrosis over the anterior wall of the left veniricle consistent with old healed infarction. There were no mural thrombi or other changes in the heart. The carotid and vertebral arteries in the neck showed only a mild non-occlusive atheroma, as did the main aorta. The arteries in the limbs showed minimal atheroma. The lungs showed confluent bronchopneumonia and several small micro-abscesses which were confirmed by histological examination. There was no evidence of tuberculosis. No other abnormalities were found outside the nervous system.

Brain The vessels of the circle of Willis showed patchy non-occlusive atheroma and more diffuse sclerosis involving all main vessels distal to the vertebro-basilar arteries, which showed only slight atheroma. The meninges were unremarkable. The brain weighed $1100 \mathrm{~g}$ and its external surface showed coarse granular atrophy 


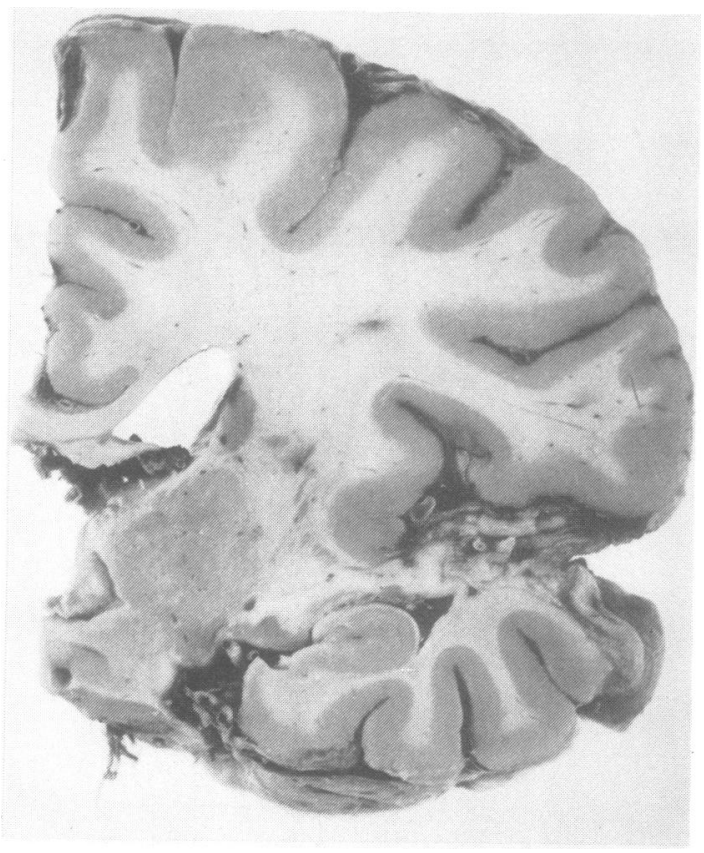

Fig 3 Right temporal lobe infarction involving cortex and white matter. Small infarcts and gliotic scars in internal capsule and central parietal white matter. Tiny apoplectic cysts (holes) in thalamus (compare with fig $2 b, c, d)$.

of both frontal poles. There were large depressed brownpigmented gliotic cortical infarcts involving the lateral surface of the right temporal and occipital lobes and the inferior surface of the left temporal lobe, including part of the hippocampal gyrus. Coronal sections through the brain confirmed that the infarct in the right temporooccipital region involved the cortex and subcortical white matter and extended into the insular cortex (fig 3) and more deeply to involve the lateral wall of the posterior horn of the right lateral ventricle, including the optic radiation and inferior longitudinal fasciculus (fig 4). On the left side cortical and subcortical infarction involved the inferior and medial surface of the temporal and occipital lobes (fig 5) and the deeper structures in the left hippocampus. In both frontal lobes there was gliosis in the white matter in front of the anterior horns of the lateral ventricles and on the left side this gliosis showed coarse cystic transformation involving an area measuring about $1.5 \mathrm{~cm}$ in cross section. At the level of the mamillary bodies close to the right caudate nucleus there was another apopleptic-type cyst measuring about $2 \times 0.5 \mathrm{~cm}$ in cross section, which extended into the internal capsule. The white matter in the corona radiata and superior longitudinal fasciculus adjacent to the right caudate nucleus also showed gliosis and cystic cavitation over a $1.5 \mathrm{~cm}$ diameter area (fig 6). There was a small cyst, about $0.3 \mathrm{~cm}$ in diameter, in the posterior part of the left thalamus. The brainstem and cerebellum were unremark-

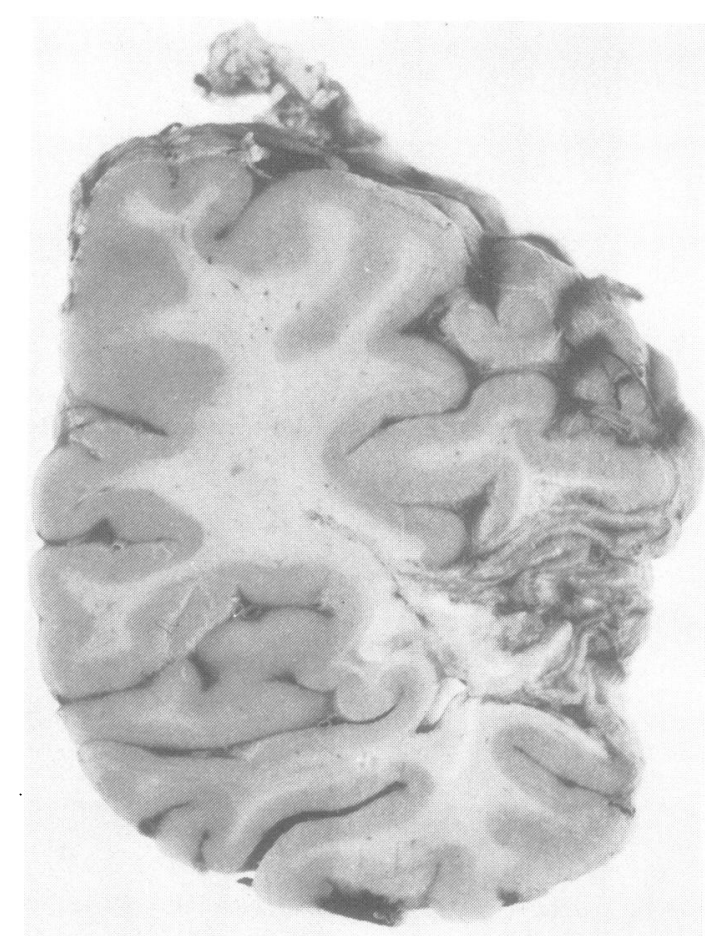

Fig 4 Extensive right temporo-occipital infarction involving cortex and visual fibres and lateral wall of posterior horn (compare with fig $2 b, c$ ).

able macroscopically.

Brain histologically Throughout the brain the large meningeal arteries showed variable degrees of adventitial and mural fibrosis, often with marked hyaline change. The small meningeal and perforating arteries showed diffuse fibrosis and hyaline adventitial and medial thickening and many were transformed into almost acellular homogenous retractile rings (fig 7). Some of these vessels also showed marked fibro-fatty intimal proliferation. There was no evidence of active or healed arteritis as evidenced by inflammatory infiltration or breaches in the elastica. The arterioles throughout the central white matter were similarly affected and this change was often associated with atrophy of the perivascular myelin and dilatation of the perivascular spaces leading to a "cribriform" appearance (fig 8). In many places small clusters of lipid and iron-containing phagocytes were seen in the dilated perivascular spaces. In the frontal cortex there was focal loss of neurons and many of those remaining appeared shrunken and stained deeply with haematoxylin. The white matter in.the frontal region tended to stain rather poorly (fig 9) and at the level of the genu of the corpus callosum there was marked vascular sclerosis and hyalinisation and intimal proliferation, associated with definite loss of periventricular 


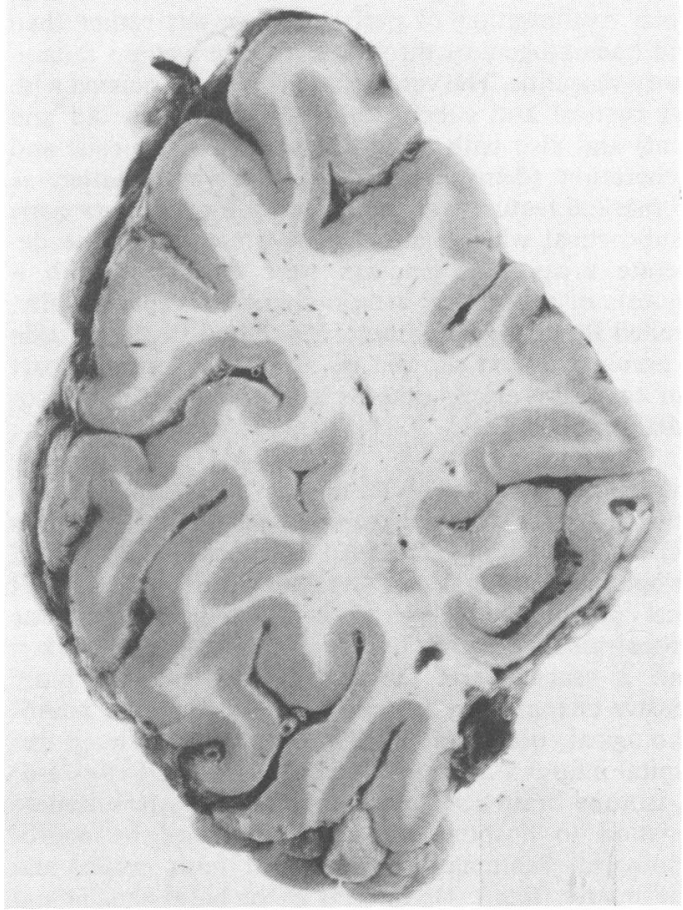

Fig 5 Old gliotic infarction of inferior surface of left occipital lobe, cribriform (perivascular) atrophy in the central white matter and tiny apoplectic cysts (compare with fig $2 a, b, c)$.

myelin. In these areas the white matter had a loose retiform appearance and showed astrocytic hyperplasia and infiltration with lipophages and siderophages. There was old perivascular infarction in the region of the head of the caudate nucleus on both sides (fig 10) with extension into the internal capsules. There was microcystic transformation in these areas associated with gliosis; confluence of microcysts had given rise to the tiny holes (apoplectic cysts) seen in figs 3,6 and 10 . Extensive infarction was also confirmed in the temporal lobes and in many places there was extension into the overlying temporal cortex which was completely disorganised. The temporal and occipital lobe lesions which involve extensively the radiation of the corpus callosum and the geniculo-calcarine radiation, were in part old and in part more recent in origin (fig 11). The older areas showed retiform and fibrillary gliosis but the more recent lesions contained abundant large lipophages and siderophages. Where the white matter showed degeneration there was a distinct tendency for the subcortical arcuate fibres to be well-preserved (figs 9-11). The pons, medulla and cerebellum showed pallor of myelin staining, extensive hyaline changes in the blood vessels and patchy loss of neurons. There were no defined infarcts in the brainstem or cerebellum. Argyrophil plaques or neurofibrillary tangles (Alzheimer changes) were not present in any area.

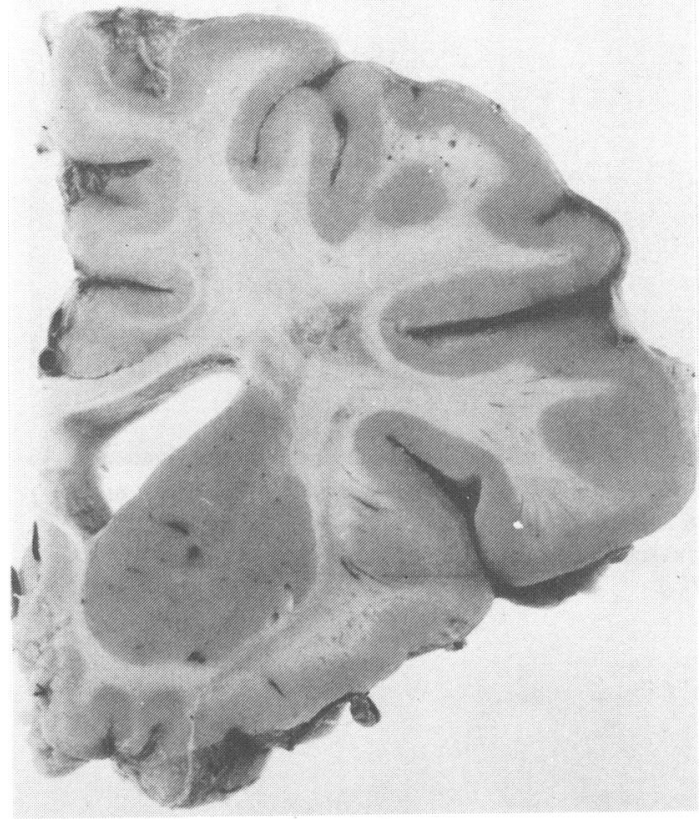

Fig 6 Gliosis and cystic cavitation in corona radiata and superior longitudinal fasciculus lateral to the angle of the body of the right lateral ventricle. Cribriform atrophy in white matter of mid-frontal gyrus and tiny apoplectic cysts in striatum (compare with figs $1 d$, and $2 d, e)$.

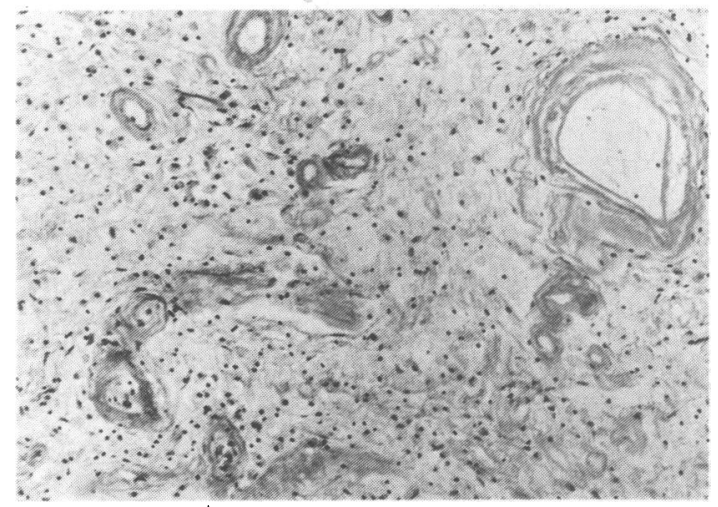

Fig 7 Concentric sclerosis and hyalinisation of small penetrating arteries in temporal white matter, marked degeneration and loss of myelin, infiltration with lipophages and early gliosis. (Haematoxylin-Van Gieson, $\times 180$.)

In summary the brain showed extensive hyaline arteriosclerosis and atheroma which diffusely affected the small intracerebral arteries and in many vessels narrowed the lumina. Overt fibrin thrombosis was not seen in these 


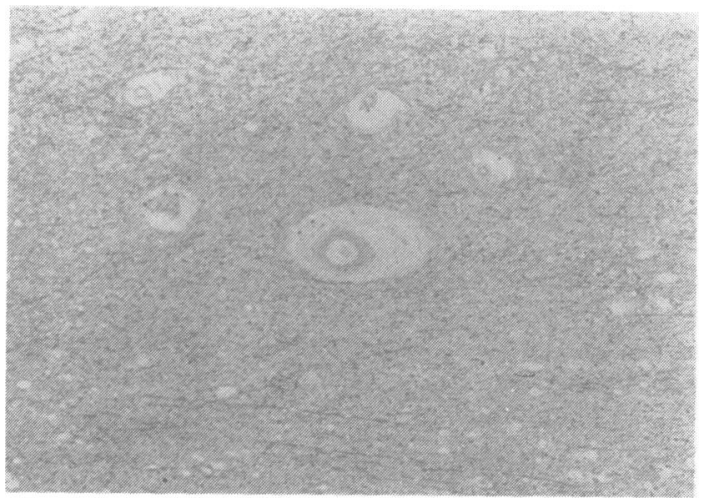

Fig 8 Pallor of myelin staining in central white matter with dilatation of perivascular spaces and atrophy of perivascular myelin. (Luxol fast blue-cresyl fast violet. $\times 72$.)

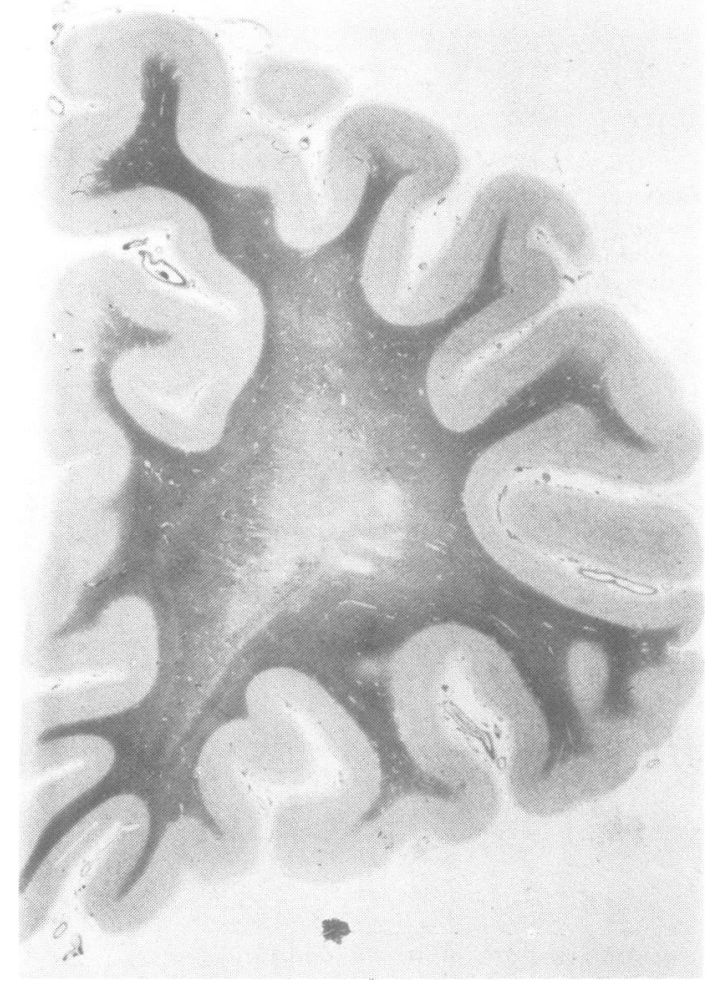

Fig 9 Degeneration of central white matter in right frontal region with cystic changes (compare with figs $1 d, e, f$, and $2 e$ ). (Luxol fast blue-cresyl fast violet.)

vessels although complete or partial occlusion by concentric mural sclerosis was often noted. The process appeared to be associated, therefore, with vascular occlusion due to progressive concentric medial and/or intimal hyalinisation of perforating vessels rather than florid haematogenous thrombosis; there was no inflammatory vasculitis. The vessel changes were associated with focal cortical and subcortical infarction (both old and recent) and also with more diffuse loss of neurons and degeneration (demyelination) of the white matter. A well-marked feature was the tendency for a narrow zone of subcortical white matter to be preserved in the degenerate areas. The findings were consistent with a diagnosis of subcortical arteriosclerotic encephalopathy, so-called Binswanger's disease. The blood vessels outside the nervous system showed no significant lesions, apart from coronary artery atherosclerosis and an old myocardial infarct.

\section{Radiological-pathological correlation}

Allowing for the different planes of sectioning, there was excellent correlation between the radiological and neuropathological findings. The infarct in the inferomedial aspect of the left temporal and occipital lobes was demonstrated by both CT scans (fig 1a-c, fig 2a-c) taken about 2 years apart; the second scan showed more extensive changes (fig $2 \mathrm{a}-\mathrm{c}$ ) corresponding to the neuropathological observations (fig 5). A right temporooccipital infarct was demonstrated by both CT (fig 2a-d) and isotope brain scan during the patient's penultimate admission to hospital and later confirmed by neuropathological examination to involve both cortex and white matter (figs 3,4 ). Infarcts in the basal ganglia and capsular areas were demonstrated by $\mathrm{CT}$ and confirmed on macroscopic and microscopic examination of the brain (figs 1d, 2c, d, 3, 6, 10). The low attenuation in frontal and parietal white matter demonstrated by the CT scans was shown neuropathologically to be due to diffuse degeneration/demyelination and cribriform perivascular atrophy of the central white matter with sparing of the subcortical U-fibres (figs 1c-g, 2d, e, f, 3, 6-11). Mild to moderate ventricular dilatation and cortical atrophy were shown by $\mathrm{CT}$ and confirmed neuropathologically.

\section{Discussion \\ th.}

The initial clinical diagnosis in this patient was thought to be multi-infarct dementia ${ }^{18}$ occurring at a young age. However, the neuropathological features which consisted of obliterative arteriosclerosis and hyalinosis of cerebral vessels, central white matter degeneration/demyelination, cortical and subcortical infarction and mild hydrocephalus are characteristic of subcortical arteriosclerotic encephalopathy. ${ }^{1151619}$ Although the extent of temporo-occipital lobe infarction ${ }^{1415}$ and luminal narrowing of pial-cortical arteries and the neuronal loss in the frontal cortex ${ }^{16}$ in this patient were unusual, they are acceptable within the definition of that disease. Similarly the basic CT scan features of this patient were those of low attenuation of the periventricular and central white matter, combined 


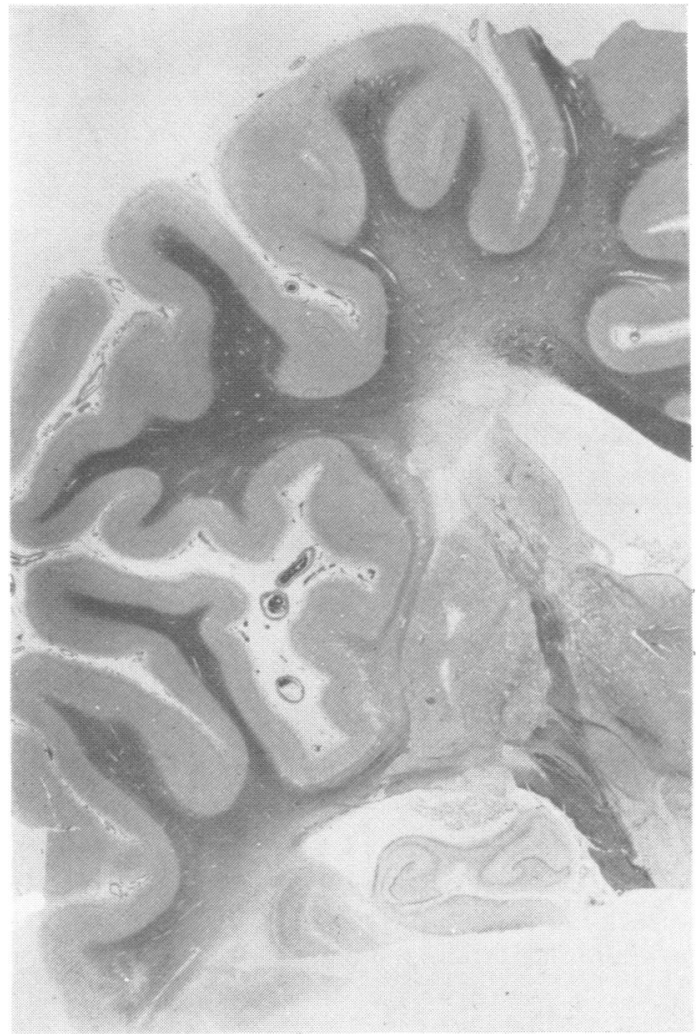

Fig 10 Infarction in region of head of left caudate nucleus and internal capsule. Small cysts in putamen. Diffuse pallor of central frontal and temporal myelin related to the lesions shown in fig 5. Widening of cerebral sulci due to cortical atrophy. (Luxol fast blue-cresyl fast violet.)

with cortical and subcortical infarcts, mild ventricular dilatation and cortical atrophy and lack of brain swelling and of contrast enhancement of the lesions; these features are compatible with subcortical arteriosclerotic encephalopathy ${ }^{2} 1617$ although large cortical infarcts are only occasionally encountered ${ }^{2} 17$ in such patients.

The salient neurological features in this patient were the recurrent and initially reversible stroke-like episodes of hemiparesis, dysphasia and confusion; episodes of loss of consciousness some of which were due to brain stem ischaemia, others to epileptic seizures; the insidious development of dementia and change in personality and finally the development of cortical blindness which was followed by accelerated mental and motor deterioration, and ultimately death 18 months later. Such a history, although unusually long (23 years) is

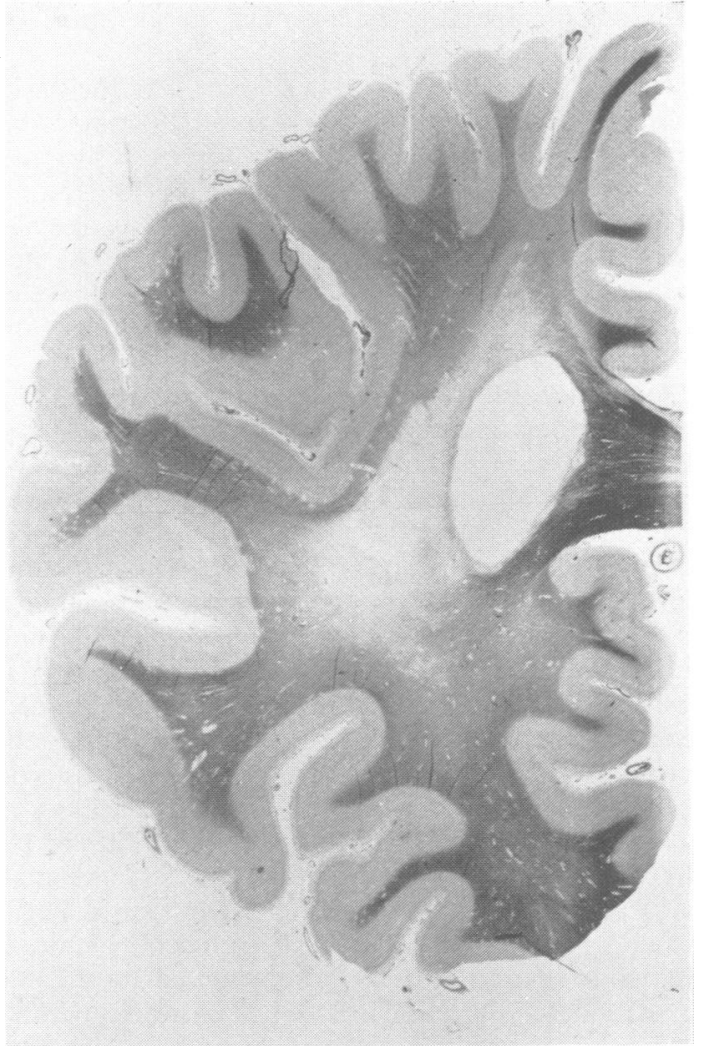

Fig 11 Severe degeneration of myelin at level of splenium of corpus callosum involving visual and callosal radiations (compare with figs $1 d$, and $2 d, e$ ). (Luxol fast blue-cresyl fast violet.)

characteristic of subcortical arteriosclerotic encephalopathy. ${ }^{1-1015-17}$ The stroke-like episodes could be explained in terms of the various subcortical infarcts demonstrated by CT as well as neuropathologically and the cortical blindness could be attributed to the bilateral temporo-occipital infarcts, occurring as a result of obliterative arterial disease. The personality changes and dementia, which from the beginning included an element of anosognosia, became apparent before the stroke-like event in 1976, when the CT scan demonstrated the first large occipitotemporal infarct. It would be reasonable to suggest, therefore, that the dementia and personality change were related to another neuropathological process, namely degeneration/demyelination of the central white matter appearing as low attenuation areas on CT. Cerebral cortical and white matter infarction in the temporal lobes (including the hippocampus) as well as neuronal loss in the frontal lobes could have 
been responsible for the terminal acceleration of dementia.

An unusual feature in this patient was the early onset of the disorder at the age of 26 years; the majority of cases described have been over 50 years of age, ${ }^{2}$ the youngest $31 . .^{15}$ In this respect the family history of deaths through strokes at a young age has special significance, though information is not available as to the co-existence of dementia and personality changes in these relatives, nor indeed on the aetiology of their strokes.

A further point of interest in this patient is the normal blood pressure and heart size. Most cases previously described have featured hypertension and indeed Caplan and Schoene ${ }^{15}$ define subcortical arteriosclerotic encephalopathy as a hypertensive vascular dementia, and others subscribe to this view. ${ }^{16}$ 20-23 However, several normotensive patients have been described including one by Caplan and Schoene. ${ }^{15}$ This suggests that the basic lesion is obliterative cerebral arteriosclerosis and that hypertension when present will further prejudice the cerebral circulation, already compromised by rigid sclerosis of small arteries.

In this patient, obliterative arteriosclerosis affected the penetrating circumferential arteries, the pialcortical vessels and the long medullary arteries, which could have resulted in chronic ischaemia of the white matter. Furthermore, such changes could have rendered the white matter and cortex vulnerable to acute hypoxaemia during episodes of syncope, hypotension or seizures, resulting in further infarction. At least one episode of stroke clearly followed myocardial ischaemia. A similar argument has been advanced by other authors ${ }^{19}$ to explain the development of hemispheric white matter degeneration, but such arguments remain speculative since cerebral blood flow studies were not carried out in this or their patients. ${ }^{19}$

Assuming that subcortical arterioscleroticencephalopathy develops more commonly in hypertensive patients, it is not clear why only a small proportion of hypertensives develop this disorder. Okeda ${ }^{12}$ found that there is a greater degree of thickening of the media of white matter arteries in subcortical arteriosclerotic encephalopathy than in hypertensive encephalopathy; he further postulated redistribution of cerebral blood flow from cerebral grey matter to white matter causing focal oedema and small vessel changes. Feigin and Popoff ${ }^{13}$ have suggested that the demyelination of subcortical arteriosclerotic encephalopathy is secondary to cerebral oedema and hypertension, rather than to changes in the blood vessel; Feigin et al $^{14}$ subsequently considered hypoxia to be an added factor. Jellinger and Neumayer ${ }^{11}$ in contrast consider that the pathogenesis of subcortical arteriosclerotic encephalopathy depends on damage to vessels coupled with a functional disturbance of the blood brain barrier and impairment of blood flow in the border zone between the penetrating arteries to the basal ganglia and the long penetrating circumferential arteries to the white matter. Crompton ${ }^{22}$ has further suggested that the cortical arteries are similarly affected, so that the white matter lesions develop as a result of end-artery ischaemia. The present case lends support to this view.

It has now become possible to define the clinical and CT feature of subcortical arteriosclerotic encephalopathy. ${ }^{215-17}$ Unusual cases such as the one describel above are recognised. Morever, studies of cerebral blood flow (and of oxygen utilisation) are feasible in a number of centres, so that patients with this disorder could be studied prospectively, and where appropriate, neuropathologically. Such studies will bridge the gap which still exists in the final definition of the syndrome of subcortical arteriosclerotic encephalopathy, and perhaps suggest therapeutic measures in addition to the control of hypertension.

We are grateful to $\mathrm{Dr} \mathrm{RJ}$ West for neuroradiological advice, Miss Mary Trumper for preparation of the histological material and Miss GL Parkinson for typing the manuscript.

\section{References}

${ }^{1}$ Olszewski J. Subcortical arteriosclerotic encephalopathy. World Neurol 1962;3:359-75.

${ }^{2}$ Loizou LA, Kendall BE, Marshall J. Subcortical arteriosclerotic encephalopathy: a clinical and radiological investigation. $J$ Neurol Neurosurg Psychiatry 1981 ;44:294-304.

3 Editorial. Binswanger's encephalopathy. Lancet 1981; $1: 923$.

- Janota I. Dementia, deep white matter damage and hypertension: "Binswanger's disease". Psychol Med $1981 ; 11: 39-48$.

${ }^{3}$ Binswanger $O$. Die Abgrenzung der allgemeinen progressiven paralyse. Berl Klin Wochenschr 1894; $31: 1103-5,1137-9,1180-6$.

- Alzheimer A. Die Seelanstörungen auf Arteriosklerotischer Grundlage. Z Psychiatr (Berl) 1902; 59:695-711.

' Nissl F. Zur Kasuistik der Arteriosklerotischen Demenz. (ein Fall von sog. "Encephalitis subcorticalis"). Z Neurol Psychiatr 1920;19:438-53.

${ }^{8}$ Farnell FJ, Globus JH. Chronic progressive vascular subcortical encephalopathy. Arch Neurol Psychiatry 1932;27:593-604.

- Davison C. Progressive subcortical encephalopathy. $J$ Neuropathol Exp Neurol 1942;1:42-8.

${ }^{10}$ Garcin R, Lapresle J, Lyon G. Encephalopathie souscorticale chronique de Binswanger. Etude anatomoclinique de trois observations. Rev Neurol (Paris) 
$1960 ; 102: 423-40$.

11 Jellinger K, Neumayer E. Progressive subcorticale vasculäre encephalopathie Binswanger. Eine klinischneuropathologische studie. Archiv Psychiatrie Zeitzchrift Neurol 1964;205:523-54.

12 Okeda R. Correlative morphometric studies of cerebral arteries in Binswanger's encephalopathy and hypertensive encephalopathy. Acta Neuropathol (Berlin) $1973 ; 26: 23-43$.

13 Feigin I, Popoff N. Neuropathological changes late in cerebral oedema: the relationship to trauma, hypertensive disease and Binswanger's encephalopathy. J Neuropathol Exp Neurol 1963;22:500-11.

14 Feigin I, Budzilovich G, Weinberg G, Ogata J. Degeneration of white matter in hypoxia, acidosis and oedema. J Neuropathol Exp Neurol 1973;32:125-43.

15 Caplan LR, Schoene WC. Clinical features of subcortical arteriosclerotic encephalopathy (Binswanger disease). Neurology (Minneap) 1978;28:1206-15.

${ }^{16}$ Rosenberg GA, Kornfeld M, Stovring J, Bicknell JM. Subcortical arteriosclerotic encephalopathy (Binswanger): computerised tomography. Neurology (Minneap) 1979;29:1102-6.
${ }^{17}$ Zeumer H, Schonsky B, Sturm KW. Predominant white matter involvement in subcortical arteriosclerotic encephalopathy (Binswanger Disease). $J$ Comput Assist Tomogr 1980;4(1):14-19.

${ }^{18}$ Hachinski VC, Lassen NA, Marshall J. Multi-infarct dementia. A cause of mental deterioration in the elderly. Lancet $1974 ; 2: 207-10$.

19 De Reuck J, Crevits L, De Coster W, Sieben G, vander Eecken $\mathbf{H}$. Pathogenesis of Binswanger chronic progressive subcortical encephalopathy. Neurology (Minneap) 1980;30:920-8.

${ }^{20}$ Burger PC, Burch JG, Kunze U. Subcortical arteriosclerotic encephalopathy (Binswanger's disease). Stroke 1976;7:626-31.

${ }^{21}$ Aronson SM, Perl DP. Clinical neuropathological conference. Dis Nerv Syst 1974 ;35:286-91.

${ }^{22}$ Crompton RM. Pathology of degenerative cerebral arterial disease. In: Ross Russell RW, ed. Cerebral Arterial Disease. Edinburgh: Churchill Livingstone, 1976:40-56.

${ }^{23}$ Gautier JC. Cerebral ischaemia in hypertension. In: Ross Russell RW, ed. Cerebral Arterial Disease. Edinburgh: Churchill Livingstone, 1976:181-209. 